\title{
Meteorosensitivity in a Group of Patients Affected by Multiple Sclerosis and Hospitalized in a Rehabilitation Facility: An Observational Study
}

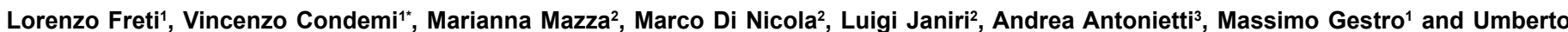
Solimene

${ }^{1}$ Department of Biomedical Sciences for Health-State University of Milan; Via Mangiagalli 31, Milan, Italy

${ }^{2}$ Istitute of Psychiatry and Clinical Psychology, Catholic University of Sacred Heart, Largo A. Gemelli 8, Rome, Italy

${ }^{3}$ Fondazione Don Gnocchi Onlus, IRCCS Santa Maria Nascente Centre; Via Alfonso Capecelatro 66, Milan, Italy

\begin{abstract}
Background: In the recent decades, the relationship between psycho-physical disorders and weather conditions has taken on an increased scientific solidity. Furthermore, it is known to every clinician that healthcare practice has an important role in the management of psycho-physical disorders of patients affected by multiple sclerosis. Given that meteorosensitivity is related to the psycho-physical feebleness, this study is an attempt to clarify if treatments in a non-acute care rehabilitation centre can reduce the meteorosensitivity in patients with multiple sclerosis.
\end{abstract}

Methods: In the spring 2014, 42 patients with MS, at Fondazione Don Gnocchi Santa Maria Nascente Centre (Milan, Italy), were observed by two evaluations (initial and final). The initial evaluation was the administration of METEO-Q, a questionnaire able to quantify the meteorosensitivity degree of the subjects, and Beck Depression Inventory-II, mainly. For each patient, passed one month, the final evaluation was carried out with the same modalities.

Results: A statistically significant difference was found between initial and final meteorosensitivity degree $(P=0.01)$, with better scores in final evaluations for most of the subjects. The correlation between Beck Depression Inventory-II and meteorosensitivity degree resulted significant and positive $(R=0.377 ; P=0.001)$ and it confirms the link between psycho-physical feebleness and meteorosensitivity. Finally, no significant correlation emerged between initial meteorosensitivity degree and body mass index $(R=0.187 ; P=0.236)$.

Conclusions: This study confirms the possibility of improving meteorosensitivity, probably by rehabilitation too. Future studies could clarify the effects of health care practice on meteorosensitivity.

Keywords: Multiple sclerosis; Rehabilitation; Biometeorology; Complementary therapies; Depression

Abbreviations: MS: Multiple Sclerosis; MTS: Meteorosensitivity or Meteorosensitive; MD: Meteorosensitivity Degree; SR: Daily Average Solar Radiation; T: Daily Average Temperature; RH: Daily Average Relative Humidity; QS: METEO-Q Scores, $\Delta$ : Variation (final value initial value).

\section{Introduction}

Multiple sclerosis (MS) is a demyelinating, chronic, inflammatory and autoimmune disease, with progressive course. It affects the white matter, and less the grey matter, in the Central Nervous System [1]. In the world, it has an incidence of 7:100.000 cases/year, each person has 1:400 chances to get it over lifetime and its onset occurs typically between 20 and 40 years old [2].

In patients with MS, a cellular and humoral immunity disorder produces disseminated injuries of nervous tissue, known as demyelinating plaques. They generally happen during critical episodes, defined as exacerbations. During an exacerbation phase, a worsening of neurological clinical picture usually occurs. In most of patients, it can regress with different degrees (remission phase) and tend to repeat over the next years. Often, remissions become smaller than exacerbations, until the worsening of neurological picture become constant [3]. It could cause different symptoms such as motor, sensory, or visual impairment, fatigue, bowel, bladder, and sexual dysfunctions [4]. MS can also determine the onset of cognitive impairments [5] and psychiatric symptoms, mainly depression [6]. For these reasons, patients with MS can be psycho-physically feeble.

Nowadays, meteorosensitivity (MTS) is no longer considered as a suggestion derived from popular myths, but it is studied by medical biometeorology. It is defined as the susceptibility of the subject to the action of one or more meteorological phenomena. They can cause physical and psychic upsets or exacerbations of preexisting symptoms. Therefore, meteorosensitive subjects reveal some difficulties to solve physical and psychic perturbations generated by physical energy of meteorological events. For these reasons, MTS can be considered as a "general adaptation syndrome", because it is linked to the daily stress management. The action of meteorological factors and other stressor events can make difficult to maintain the physiological variables balanced. When the limits of homeostatic compensation are overstepped, several somatoform disorders can develop. Therefore, the ability to manage stress represents an important prognostic factor. In fact, psycho-physical reactivity can reach a compensation state in order to successfully face meteorological stressor events [7].

Amongst the weather factors able to influence psyche, brightness is the most studied. It influences the vivacity of psycho-physical reactions by conditioning specific neuroendocrine mechanisms

*Corresponding author: Vincenzo Condemi, Department of Biomedical Sciences for Health-State University of Milan; Via Mangiagalli 31, 20133-Milan, Italy, Tel: +390250318460; E-mail: vincenzo.condemi@unimi.it

Received October 17, 2017; Accepted October 23, 2017; Published October 30 2017

Citation: Freti L, Condemi V, Mazza M, Nicola MD, Janiri L, et al. (2017) Meteorosensitivity in a Group of Patients Affected by Multiple Sclerosis and Hospitalized in a Rehabilitation Facility: An Observational Study. Altern Integ Med 6: 252. doi: 10.4172/2327-5162.1000252

Copyright: (c) 2017 Freti L, et al. This is an open-access article distributed under the terms of the Creative Commons Attribution License, which permits unrestricted use, distribution, and reproduction in any medium, provided the original author and source are credited. 
Citation: Freti L, Condemi V, Mazza M, Nicola MD, Janiri L, et al. (2017) Meteorosensitivity in a Group of Patients Affected by Multiple Sclerosis and Hospitalized in a Rehabilitation Facility: An Observational Study. Altern Integ Med 6: 252. doi: 10.4172/2327-5162.1000252

Page 2 of 8

involved in circadian and circannual rhythms. The two last editions of the Diagnostic and Statistical Manual of Mental Disorders recognize a disorder in which people become depressed in a certain period of the year, such as winter and summer, the Seasonal Affective Disorder (SAD) [8]. SAD seems to be related to latitude [9], in fact one of the causes of $\mathrm{SAD}$ is the variation in hours of daily sunshine [10]. Moreover, several trials show an efficacy of bright light on depressive symptomatology in non-seasonal depression [11]. Weather factors seem to be related also to both attempted and completed suicides with a non-seasonal pattern [12]. Other conditions, such as anxiety [13], concentration reductions [14] and aggressions [15] seem to show a significant meteorotropism.

The other strongest evidences regard the combined action of high and low temperatures and humidity on cardiovascular and musculoskeletal systems $[16,17]$.

Therefore, it is appropriate to consider patients with MS as particularly meteorosensitive subjects and to choose MS for the study of MTS.

Patients affected by MS are susceptible especially to relatively high temperature. This condition is known as Uhthoff's phenomenon. It is a disorder of neurological functions that is stereotyped, short in duration (less than 24 hours), reversible and related to recurrent fluctuations in axonal conduction properties. These acute changes can be linked to various antecedent triggering factors, all of which are associated in some way with an increase in core body temperature. The main causal factors are high environmental temperature, fever, physical exercise, hot baths or showers, increase of psychological stress and perimenstrual period [18].

Medical Biometeorology's approach to disease is the same as Eastern medicine [19]. As explained previously, right mental behaviours can avoid a psycho-physical imbalance, reach a compensation state in order to successfully face stressor events and consequently avoid physical disease. That is why psychological assistance and/or meditation are essential for the eradication of meteorosensitivity. Some symptomatological therapies are also possible and already adopted. They are physical and bioenergetics techniques; Reiki, acupuncture, homeopathy and phytotherapy are peculiar examples. In different ways, they act by stimulating the physiological organic reactions [20].

Moreover, climatotherapy, that uses the positive action exercised by the climate of specific places, could represent an option to be investigated better.

Finally, healthcare practice has also an important role in the management of psycho-physical disorders of patients [21]. Therefore, it is logical to wonder whether the clinical-rehabilitation practice can represent a symptomatological care for meteorosensitivity. The main purpose of this study is to provide some clarifications of this issue, taking in exam a sample of patients affected by multiple sclerosis.

\section{Material and Methods}

\section{Subjects}

Patients with MS flocking to Fondazione Don Gnocchi, Santa Maria Nascente Centre (Milan-Italy) were selected according to specific inclusion/exclusion criteria. After the inclusion/exclusion procedures, 42 patients (female $=28 \%$ to $67.7 \%$; male $=14 \%$ to $33.3 \%$ ) were observed. In this rehabilitation centre, generally people are recovered with no exacerbation of disease in course. Therefore, their permanence in the structure could be also a temporary interruption of their chaotic race of everyday life, because they receive assistance from the health workers and socialize with other people with MS.

\section{Inclusion/exclusion criteria}

The following patients with MS were included in the study:

a) with no current exacerbation, for assessing only people with a stable clinical condition;

b) regardless the clinical picture (Kurtzke's EDSS and MS type), because, given the study protocol and methods, they were not considered as important elements;

c) with a normal adjusted score $(\geq 24)$ of Mini Mental State Examination (MMSE), Measso et al. version (22), for selecting only people able to answer suitably to questionnaires;

d) whose treatment cycle lasted for at least a month;

e) that finished the previous rehabilitation cycle for more than one month before the initial evaluation. Thus, this period can be compared with the observation month

\section{Evaluation scales}

For patient inclusion and exclusion, MMSE, Measso et al. [22] version was employed.

For patient evaluation, the following scales were employed:

a) Beck Depression Inventory-II (BDI-II), for the evaluation of depressive symptoms and behavioural characteristics of depression $[23,24]$

b) METEO-Q questionnaire, for establishing the meteorosensitivity degree (MD) of the subject (Questionnaire 1). It is a selfassessment questionnaire recently developed and validated with an excellent internal consistency: Cronbach's $\alpha$ (items 1-5) $=0,81$ and Cronbach's $\alpha$ (items 6-11) $=0,87$ [25]. It is composed of two groups of items (1-5 and 6-11) and one checklist. Each group of items, independently from the other, allows determining the MD of the subject. The only other available statistic about METEO-Q is an accuracy of 0,73 in a sample of bipolar subjects and 0,68 in a sample of healthy subjects [26]. The METEO-Q questionnaire analyses all the main symptoms usually correlated to weather/ climatic conditions. In fact, this study does not examine a specific symptom, even if patients with MS usually refer some typical upsets.

\section{Study protocol}

In the spring 2014, each admitted patient was included/excluded according to the inclusion/exclusion criteria. On the day of his entry, each patient was evaluated (initial evaluation). The initial evaluation was:

a) Administration of BDI-II;

b) Administration of METEO-Q;

c) Registration of subjects' height and weight, used for the body mass index (BMI) calculation.

After one month from initial evaluation, the final one was carried out with the same modalities. About the administration modalities of METEO-Q, some clarifications (specific of this case) need to be done. In METEO-Q instructions, there are not specific indications about a temporal parameter to which patient should refer to during the answers. Therefore, a time frame of 1 month was chosen: patients 


\section{METEO-Q questionnaire \\ (Mazza M., Di Nicola M., Janiri L.)}

Name.

Date of Birth.

Date of Evaluation.

For every item attribute a score as the following legend:

$0=$ absent, $1=$ light, $2=$ moderate, $3=$ considerable, $4=$ serious.

1) Variations of mood in relation to the change of latitude, the geographic zone, the jet leg.

Specify induced modifications...

2) Variations of mood in relation to atmospheric changes (ex.: when it begins or when

it stops raining, when it is cloudy, when it is sunny or when the sun suddenly goes

down, when humidity increases).

Specify induced modifications.

3) Variations of mood in relation to the brightness of the sky (when days are more or less luminous or when days "grow tall" or "they are shortened" according to the seasons).

Specify induced modifications.

4) Variations of mood caused by the temperature changes (warmer or colder days).

Specify induced modifications.

5) Mood changes caused by the seasons changing.

Specify induced modifications...

6) Indicate the degree of relation between the set in symptomatology and the climatic or atmospheric change.

7) Tendency of these disturbs to minimize or disappear when the triggering condition stops or when an opposite environmental condition comes up.

8) Eventual coincidence of these disturbs with other cyclical phenomena (ex.: menstrual cycle).

9) Presence of prodromical symptoms few days before the climatic modifications (irritability, weariness...).

10) Interference with daily activities due to disturbs induced by climatic changes.

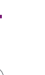




\section{Metereopathy Checklist}

Indicate the disturbs related to meteorological changes that you mostly frequently experience. For every voice mark: absent $=0$, light $=1$, moderate $=2$, considerable $=3$, severe $=4$.

- lability of mood;

$\begin{array}{lllll}0 & 1 & 2 & 3 & 4\end{array}$

(oscillations of mood within a day or few more days)

- extreme reactivity to external events;

$\begin{array}{lllll}0 & 1 & 2 & 3 & 4\end{array}$

- depression;

$\begin{array}{lllll}0 & 1 & 2 & 3 & 4\end{array}$

- anxiety;

$\begin{array}{lllll}0 & 1 & 2 & 3 & 4\end{array}$

- asthenia;

$\begin{array}{lllll}0 & 1 & 2 & 3 & 4\end{array}$

- lack of appetite, anhedonia;

$\begin{array}{lllll}0 & 1 & 2 & 3 & 4\end{array}$

- irritability, nervousness;

$\begin{array}{lllll}0 & 1 & 2 & 3 & 4\end{array}$

- indefinite feeling of malaise;

$\begin{array}{lllll}0 & 1 & 2 & 3 & 4\end{array}$

- vague pain, articular pain, muscular pain ;

$\begin{array}{lllll}0 & 1 & 2 & 3 & 4\end{array}$

- vertigos;

$\begin{array}{lllll}0 & 1 & 2 & 3 & 4\end{array}$

- headache;

$\begin{array}{lllll}0 & 1 & 2 & 3 & 4\end{array}$

- nausea;

$\begin{array}{lllll}0 & 1 & 2 & 3 & 4\end{array}$

- alterations of the cardiac rhythm (tachycardia);

$\begin{array}{lllll}0 & 1 & 2 & 3 & 4\end{array}$

- difficulty with concentration;

$\begin{array}{lllll}0 & 1 & 2 & 3 & 4\end{array}$

- insomnia;

$\begin{array}{lllll}0 & 1 & 2 & 3 & 4\end{array}$

- excessive sleepiness;

$\begin{array}{lllll}0 & 1 & 2 & 3 & 4\end{array}$

- lack of appetite;

$\begin{array}{lllll}0 & 1 & 2 & 3 & 4\end{array}$

- excessive appetite;

$\begin{array}{lllll}0 & 1 & 2 & 3 & 4\end{array}$

- digestion's disfunctions;

$\begin{array}{lllll}0 & 1 & 2 & 3 & 4\end{array}$ (meteorism, belches, acidity, flatulence, irritable colon)

- alterations of sexuality;

(lack of desire or on the contrary hyperactivity)

$\begin{array}{lllll}0 & 1 & 2 & 3 & 4\end{array}$

- weakness during working activities.

$\begin{array}{lllll}0 & 1 & 2 & 3 & 4\end{array}$

Other:

$\begin{array}{lllll}0 & 1 & 2 & 3 & 4\end{array}$ 
TOTAL SCORE ITEMS 1-5: $\mathrm{x}=$

TOTAL SCORE ITEMS 6-11: $\mathrm{x}=$

\section{CUT-OFF SCORES}

Male

\begin{tabular}{|c|c|c|c|c|}
\cline { 2 - 5 } \multicolumn{1}{c|}{} & $\begin{array}{c}\text { Low } \\
\text { sensitivity }\end{array}$ & $\begin{array}{c}\text { Medium } \\
\text { sensitivity }\end{array}$ & $\begin{array}{c}\text { High } \\
\text { sensitivity }\end{array}$ & Meteoropathy \\
\hline Items 1-5 & $\mathrm{x}<2$ & $3<\mathrm{x}<5$ & $6<\mathrm{x}<8$ & $\mathrm{x}>9$ \\
\hline Items 6-11 & $\mathrm{x}<2$ & $3<\mathrm{x}<4$ & $5<\mathrm{x}<7$ & $\mathrm{x}>8$ \\
\hline
\end{tabular}

Female

\begin{tabular}{|l|c|c|c|c|}
\cline { 2 - 5 } \multicolumn{1}{c|}{} & $\begin{array}{c}\text { Low } \\
\text { sensitivity }\end{array}$ & $\begin{array}{c}\text { Medium } \\
\text { sensitivity }\end{array}$ & $\begin{array}{c}\text { High } \\
\text { sensitivity }\end{array}$ & Meteoropathy \\
\hline Items 1-5 & $\mathrm{x}<4$ & $5<\mathrm{x}<7$ & $8<\mathrm{x}<10$ & $\mathrm{x}>11$ \\
\hline Items 6-11 & $\mathrm{x}<3$ & $4<\mathrm{x}<6$ & $7<\mathrm{x}<10$ & $\mathrm{x}>11$ \\
\hline
\end{tabular}

Questionnaire 1: METEO-Q questionnaire and its instructions and cut-off scores.

were asked to refer to their condition of the previous month. This has the aim of making the initial and final METEO-Q scores comparable. Furthermore:

a. Items 1-5 were not administered, because they are related to conditions considered unchangeable during the interval between initial and final evaluations;

b. As explained previously, items 6-11 are sufficient for assessing MTS;

c. Patients tried to evaluate especially air temperature, solar radiation and humidity influences. This choice is explained in the following paragraph.

During initial and final evaluations, patient's treatments were not influenced.

Once collected all data, the necessary statistical calculations were performed (see "Statistical methods").

Because initial evaluations were not carried out all on the same day, the subjects have not been exposed precisely to the same weather factors and this is a methodological problem. This point was partially engaged by considering some meteorological data (revealed by the State University of Milan's meteorological station) in the statistical methodology.

\section{Climatological database and geo-demographic characteristics}

The climatological database was released by State University of Milan from February to June 2014. The following meteorological parameters were considered: solar radiation (SR), daily average temperature $(\mathrm{T})$ and relative humidity $(\mathrm{RH})$, because they are the only ones that can influence also the indoor environment of the structure that is devoid of air conditioning.

Milan $\left(45^{\circ} 27^{\prime} 50.98^{\prime \prime} \mathrm{N} 9^{\circ} 11^{\prime} 25.21^{\prime \prime} \mathrm{E}\right)$, the second-most populous city in Italy, serves as the capital of Lombardy. Milan's climate is similar to much of northern Italy's inland plains, where hot, sunny summers and moderately cold, wet and foggy winters prevail. The spring season (March-May) is characterized by strong weather variability: in March it may happen some snow, while in May temperatures $>30^{\circ} \mathrm{C}$ are possible.

\section{Statistical methods}

Preliminarily, the Shapiro-Wilk test was applied with the aim of verifying the normality of distributions. After having pointed out the non-normality of data distributions, non-parametric tests were chosen to verify if:

a. a significant difference between initial and final MD was present (intra-group difference);

b. a significant correlation between BDI-II scores and MD was present;

c. a significant correlation between initial MD and BMI was present.

The answer to question (a) represents the main purpose of this study.

Questions (b) and (c) were engaged with the aim of providing some additional data on MTS study.

To answer question (a), Wilcoxon signed-ranked test was applied. In this case the comparison was made between initial and final METEO-Q scores (QS). To answer questions (b) and (c), Spearman's rank correlation coefficient was applied. For question (b) the Spearman's 
Citation: Freti L, Condemi V, Mazza M, Nicola MD, Janiri L, et al. (2017) Meteorosensitivity in a Group of Patients Affected by Multiple Sclerosis and Hospitalized in a Rehabilitation Facility: An Observational Study. Altern Integ Med 6: 252. doi: 10.4172/2327-5162.1000252

Page 6 of 8

rank correlation coefficient application was carried out without discerning between initial and final evaluation. Instead, for question (c) it was carried out between initial QS and the BMI of each patient.

The same test was employed for engaging the meteorological matter previously described in "Study protocol". Through Spearman's rank correlation coefficient, the MD variation ( $\Delta=$ final score-initial score) was compared with $\Delta \mathrm{T}$ and $\Delta \mathrm{SR}$. In so doing, it is possible to obtain a clue about if $\Delta \mathrm{MD}$ is due to the health care practice, rather than the passage to a more favourable meteorological condition. $\mathrm{RH}$ was not considered in statistical methodology, because it has not an approximately linear trend over time (see "4. Discussion”).

All statistical significance levels were posed to $\mathrm{p}=0.05$ and all confidence intervals to $95 \%$.

For Shapiro-Wilk test application the statistical software $\mathrm{R}$ was employed. Descriptive statistics calculations and the several nonparametric tests were carried out through the statistical software SPSS.

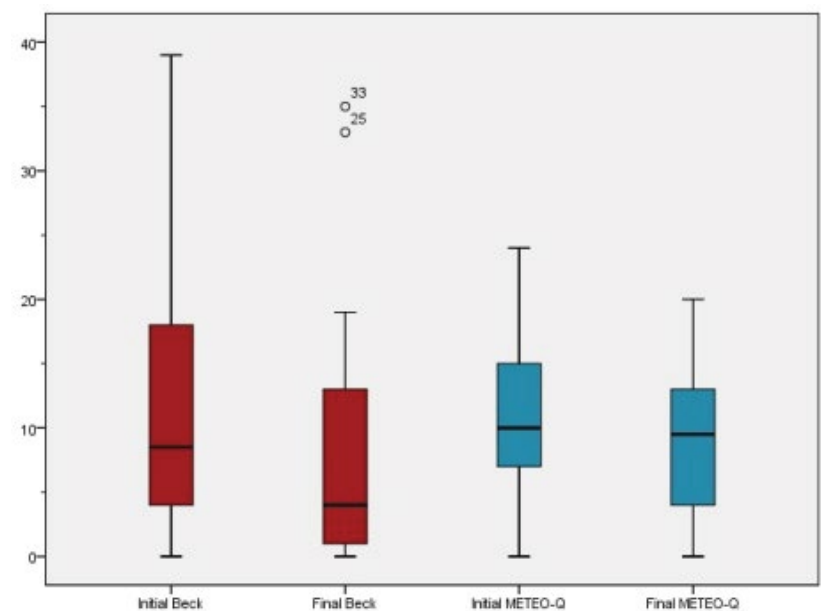

Figure 1: Boxplots constructed with raw data, both initial and final, obtained by the administration of the evaluation scales to the patients.

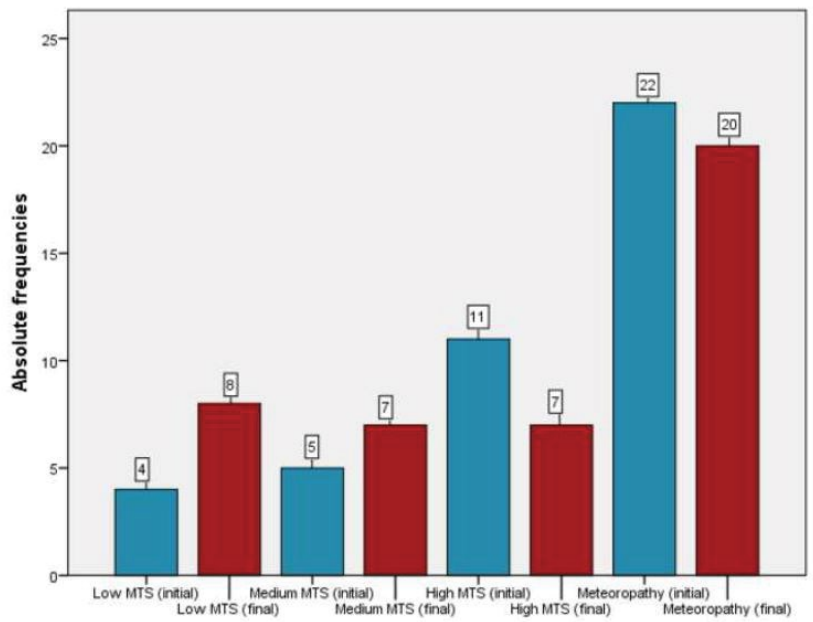

Figure 2: Absolute frequencies of the several METEO-Q cut off scores. The blue bars represent initial evaluations, while the red bars the final ones.

\section{Results}

According to the position and distribution measures and to the analysis of frequencies, it is possible to assert that a general decrease of all evaluation scores occurred. Graphic visualization of data distributions is possible by observing Figure 1. How data frequencies related to METEO-Q varied passing from initial to final evaluation is represented in Figure 2. After Wilcoxon signed-ranked test application, a statistically significant difference was observed $(\mathrm{p}=0.01)$.

Through Spearman's rank correlation coefficient, the only statistically significant correlation observed was between BDI-II and MD ( $\mathrm{R}=0.377 ; \mathrm{p}=0.001)$. Instead, as shown in Table 1 , the other correlations were not statistically significant. Finally, Figure 3 shows the trend of daily $\mathrm{T}$ and SR passing from $1^{\text {st }}$ February to $30^{\text {th }}$ June 2014. In this figure RH was not considered because it has constant daily variation.

\begin{tabular}{|c|c|c|}
\hline Comparisons & $\mathbf{R}$ & $\mathbf{p}$ \\
\hline $\mathrm{MD}$ and BDI-II & 0.377 & $0.001^{* *}$ \\
\hline $\mathrm{MD}$ and B.M.I. & 0.187 & 0.236 \\
\hline$\Delta \mathrm{MD}$ and $\Delta \mathrm{T}$ & -0.155 & 0.327 \\
\hline$\Delta \mathrm{MD}$ and $\Delta \mathrm{SR}$ & 0.063 & 0.693 \\
\hline
\end{tabular}

*: correlation is significant to level 0.05 (two tails); **: correlation is significant to level 0.01 (two tails)

Table 1: Rho correlation coefficients $(R)$ and $p$-values $(p)$ resulted from the comparisons between the variables considered.
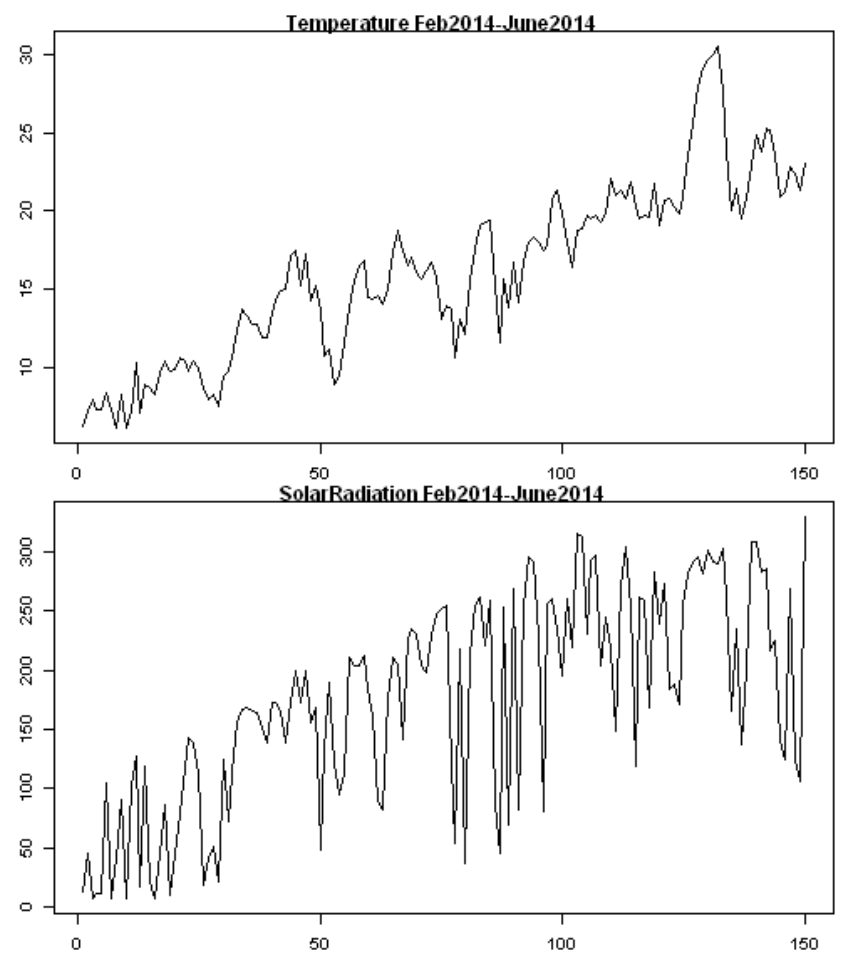

Figure 3: The trend of daily $T$ and SR passing from $1^{\text {st }}$ February to $30^{\text {th }}$ June 2014 
Citation: Freti L, Condemi V, Mazza M, Nicola MD, Janiri L, et al. (2017) Meteorosensitivity in a Group of Patients Affected by Multiple Sclerosis and Hospitalized in a Rehabilitation Facility: An Observational Study. Altern Integ Med 6: 252. doi: 10.4172/2327-5162.1000252

Page 7 of 8

\section{Discussion}

The observed decrease of BDI-II scores is an index of depression improvement in most of the patients. Final QS also were resulted generally lower than the initial ones. In fact, the correlation between BDI-II scores and MD was resulted positive $(\mathrm{R}>0)$ and statistically significant. It means these variables are related: according to this result, considering MTS in conjunction with psycho-physical feebleness seems to be suitable.

The main question of this study is the first one posed. The difference between initial and final QS was resulted statistically significant. It means that MTS improvement was not due to chance. However, it is not possible to precisely establish to what factor/s it is imputable to, because of the absence of a control group in the statistical methodology and the difficulty to exclude that it is due to the passage to a more favorable meteorological condition. Given that no significant correlation about the meteorological issue resulted, there is a tendency to think that, in this specific case, the influence on MD is due more to health care practice. However, the control group is essential for more suitable results. This thought can be extended with more certainty only to $\mathrm{T}$ and SR, whose values increased nearly linearly passing from March to May in Milan (Figure 3).

The last comparison was not resulted statistically significant. Considering this sample, BMI does not seem to be related to MD. More studies are necessary; in fact the excessive or insufficient BMI indicates a psycho-physical feebleness and therefore MTS.

These are reflections on results obtained in a study for which some methodological limits are present. They are due to the impossibility:

a. to perform a blind or a double-blind study;

b. to consider patient's memory and perceptions surely reliable;

c. to consider bigger samples of patients;

d. to consider daily variation of meteorological variables;

e. to consider the micro-climate indoor conditions (even if air conditioning was not present).

\section{Conclusions}

The present study demonstrates that MTS is a modifiable condition, maybe through health care practice.

For this practice the approach to patient is crucial too. The official medicine tends to consider the human being in his physical part and it gives no relevance to his psychic and moral aspects. This vision could not be enough in treating several pathologies, such as some psychosomatic diseases or MTS itself. They need a different approach, which considers also the action of the psyche on the human body. In order to ensure a more complete attention to the patient, our suggestion to health professionals, although into the limit of own professional competences and complementarily to the traditional cares, is to educate patients to acquire more correct mental approaches towards several life situations. In other words, our suggestion is to abide by bio-psychosocial model, based as much as possible on collaborative care. The health care professional should be more aware about the connections between mind and body and became a guide for those patients who want to find their road to a life of wealth. This approach should be adopted in future studies on MTS, for which this study could represent a starting point. Considering the methodological limits previously cited, further studies are necessary with the aim of clarifying the effects of health care practice on MTS, for example taking in exam some bigger groups of patient and a control group not subject to any treatment.

\section{Acknowledgments}

We thank Fondazione Don Gnocchi, Santa Maria Nascente Center, especially Dr. Angelo Montesano's Service of Outpatient Rehabilitation and Dr. Marco Rovaris's Unit of Neuromotor Rehabilitation and Multiple Sclerosis Centre, for the availability and support they shown us. For the same reasons we also thank the participants involved in this study.

\section{Formatting or funding sources}

This research did not receive any specific grant from funding agencies in the public, commercial or not-for-profit sectors.

\section{Ethics approval and consent to participate}

All procedures performed in studies involving human participants were in accordance with the ethical standards of the institutional and/or national research committee and with the 1964 Helsinki declaration and its later amendments or comparable ethical standards. This article does not contain any studies with animals performed by any of the authors.

\section{References}

1. Compston A, Coles A (2002) Multiple sclerosis. Lancet 359: 1221-1231.

2. Weinshenker BG, Bass B, Rise GP, Noseworthy J, Carriere W, et al. (1989) The natural history of multiple sclerosis: a geographically based study, 2. Predictive value of the early clinical course. Brain 112: 1419-1428.

3. Confavreux C, Vukusic S, Moreau T, Adeleine P (2000) Relapses and progression of disability in multiple sclerosis. New Engl J Med 343: 1430-1438.

4. Nomjooyan F, Ghanavati R, Majdinasab N, Jokari S, Janbozorgi M (2014) Uses of complementary and alternative medicine in multiple sclerosis. J Tradit Complement Med 4: 145-152.

5. Schoonheim MM, Meijer KA, Geurts JJ (2015) Network collapse and cognitive impairment in multiple sclerosis. Front Neurol 6: 82.

6. Sarısoy G, Terzi M, Gümüş K, Pazvantoğlu O (2013) Psychiatric symptoms in patients with multiple sclerosis. Gen Hosp Psychiat 35: 134-140.

7. Solimene U, Brugnoli A (2000) Meteorologia e climatologia medica. Tempo clima e salute. Mediamed, Rome.

8. American Psychiatric Association (2013) Diagnostic and Statistical Manual of Mental Disorders $\left(5^{\text {th }}\right.$ edn $)$ American Psychiatric Association, Washington.

9. Kegel M, Dam H, Ali F, Bjerregaard P (2009) The prevalence of seasona affective disorder (SAD) in Greenland is related to latitude. Nord J Psychiat 63: 331-335

10. McWilliams S, Kinsella A, O'Callaghan E (2014) Daily weather variables and affective disorder admissions to psychiatric hospitals. Int J Biometeorol 58 : 2045-2057.

11. Martiny K (2004) Adjunctive bright light in non-seasonal major depression. Acta Psychiat Scand 425: 7-28.

12. Deisenhammer EA (2003) Weather and suicide: The present state of knowledge on the association of meteorological factors with suicidal behaviour. Acta Psychiat Scand 108: 402-409.

13. Bulbena A, Pailhez G, Aceña R, Cunillera J, Rius A, et al. (2005) Panic anxiety under the weather? Int J Biometeorol 49: 238-243.

14. Pelagatti MM, Fuà D, Galliani C, Condemi V (2006) Statistical investigation on the relation between car accidents and warm katabatic winds. Nuovo Cimento C 29: 229-235

15. Butke P, Sheridan SC (2010) An analysis of the relationship between weather and aggressive crime in Cleveland, Ohio. Weather, Climate, and Society 2 127-139.

16. Yu W, Mengersen K, Wang X, Ye X, Guo Y, et al. (2012) Daily average temperature and mortality among the elderly: A meta-analysis and systematic review of epidemiological evidence. Int J Biometeorol 56: 569-581.

17. Strusberg I, Mendelberg RC, Serra HA, Strusberg AM (2002) Influence of weather conditions on rheumatic pain. J Rheumatol 29: 335-338.

18. Frohman TC, Davis LS, Beh S, Greenberg MB, Remington G, et al. (2013) 
Citation: Freti L, Condemi V, Mazza M, Nicola MD, Janiri L, et al. (2017) Meteorosensitivity in a Group of Patients Affected by Multiple Sclerosis and Hospitalized in a Rehabilitation Facility: An Observational Study. Altern Integ Med 6: 252. doi: 10.4172/2327-5162.1000252

Page 8 of 8

Uhthoff's phenomena in MS-clinical features and pathophysiology. Nat Rev Neurol 9: 535-540.

19. Cheng YC (2015) Opportunities for traditional Chinese medicine to address unmet challenges in modern healthcare. J Tradit Complement Med 7: 2-4.

20. Solimene U, Brugnoli A, Minelli E (2002) Meteoropatie. Le condizioni atmosferiche che influiscono sulla salute e sull'umore. Red edizioni, Novara.

21. Van der Ploeg HP, Streppel KR, van der Beek AJ, van der Woude LH, van Harten WH, et al. (2008) Underlying mechanisms of improving physical activity behavior after rehabilitation. Int J Behav Med 15: 101-108.

22. Measso G, Cavarzeran F, Zappalà G, Barry DL, Thomas HC, et al. (1993) The mini mental state examination: Normative study of an Italian random sample. Dev Neuropsychol 9: 77-85.

23. Beck AT, Brown GK, Steer RA (1996) Manual for the Beck Depression Inventory-II. The Psychological Corporation, San Antonio.

24. http://www.rehabmeasures.org/Lists/RehabMeasures/PrintView.aspx?ID=904

25. Mazza M, Di Nicola M, Catalano V, Callea A, Martinotti G, et al. (2012) Description and validation of a questionnaire for the detection of meteoropathy and meteorosensitivity: The METEO-Q. Compr Psychiat 53: 103-106.

26. Janiri L, Spinetti G, Mazza M, Di Nicola M (2009) Meteoropathy. A new disease. In: Christodoulou G, Jorge M, Mezzich J, editors. Advances in Psychiatry. Beta Medical Publishers, Athens, p. 45-52. 